\title{
DETERMINAÇÃO DE RESÍDUOS DOS AGROTÓXICOS TIAMETOXAM, BIFENTRINA E PERMETRINA EM AMOSTRAS DE SOLO
}

\author{
IRZO ISAAC ROSA PORTILHO* \\ RÔMULO PENNA SCORZA JÚNIOR ** \\ ELIANA FREIRE GASPAR DE CARVALHO DORES ***
}

\begin{abstract}
Métodos analíticos para a quantificação dos resíduos de tiametoxam, bifentrina e permetrina em solo usando cromatografia a líquido de alta eficiência foram desenvolvidos e validados. Diferentes métodos e solventes para a extração dos três inseticidas foram testados em solos com teor de matéria orgânica entre 32,8 e $81,7 \mathrm{~g} \mathrm{dm}^{-3}$. O método utilizando agitação por uma hora e solução extratora com a mistura acetona + diclorometano (1:1) mostrou-se o mais adequado. Obtiveram-se recuperações médias de a $89,6 \%( \pm 3,7)$ para tiametoxam, 100,5 \% ( $\pm 4,1)$ para permetrina e $92,5 \%( \pm 4,5)$ para bifentrina, com coeficientes de variação inferiores a $14 \%$ para todos os inseticidas. Os limites de detecção para tiametoxam, bifentrina e permetrina foram $0,07,0,05$ e $0,05 \mu \mathrm{g} \mathrm{g}^{-1}$, respectivamente, com limite de quantificação para todos inseticidas de $0,25 \mu \mathrm{g} \mathrm{g}^{-1}$.
\end{abstract}

PALAVRAS-CHAVE: CLAE; SOLO; PERMETRINA; BIFENTRINA; TIAMETOXAM; RESÍDUOS DE AGROTÓXICOS.

* Biólogo, doutorando em Recursos Naturais, Universidade Estadual de Mato Grosso do Sul (UEMS), Dourados, MS, Brasil (e-mail: irzo_i@terra.com.br).

** Engenheiro Agrônomo, Ph.D. em Ciências Ambientais, Pesquisador da Embrapa Agropecuária Oeste, professor, Curso de Pós-graduação em Recursos Naturais, UEMS, Dourados, MS, Brasil (e-mail: romulo. scorza@embrapa.br).

*** Engenheira Química, Doutora em Química, professora, Programa de Pós-graduação em Recursos Hídricos, Universidade Federal de Mato Grosso (UFMT), Cuiabá, MT, Brasil (e-mail: eliana@ufmt.br). 


\section{INTRODUÇÃO}

O uso de agrotóxicos na agricultura brasileira tem garantido patamares elevados de produtividade e rendimento econômico em função do controle mais eficiente de pragas, doenças e plantas daninhas. Por outro lado, a presença de resíduos dos agrotóxicos no solo, água e ar caracteriza a contaminação desses compartimentos ambientais (SCHEYER et al., 2005; MOREIRA et al., 2012; NOGUEIRA et al., 2012). Diante disso, há necessidade de estudos que monitorem a presença dos agrotóxicos principalmente no solo onde, na maioria das situações, são inicialmente depositados (CHAIM et al., 1999). Para tal, é importante o desenvolvimento e adaptação de métodos analíticos para identificação e quantificação de resíduos de agrotóxicos no solo. Entre as dificuldades que envolvem as análises desses resíduos em matrizes sólidas como o solo tem-se a sua complexidade, tempo de extração e a quantidade de solventes utilizada (ANDREU e PICÓ, 2004). Métodos com uso de ultrassom e purificação por meio de extração em fase sólida podem apresentar características de rapidez, economia, robustez e segurança (GONÇALVES ALPENDURADA, 2005; LEHOTAY et al., 2005).

A Cromatografia a Líquido de Alta Eficiência (CLAE) tem se destacado na química analítica por apresentar vantagens na análise de amostras com polaridades variadas numa única corrida (LANÇAS, 2009). Estudos com o objetivo de validação de métodos analíticos para extração simultânea de compostos com diferentes polaridades são escassos. Esses métodos são importantes já que num único sistema de produção agrícola e mesma safra pode ocorrer a aplicação de vários agrotóxicos com polaridades diferenciadas. Além disso, o método deve ser robusto para a extração desses resíduos em solos com diferentes teores de matéria orgânica (MO) devido, por exemplo, a incorporação diferenciada de resíduos vegetais. O maior teor de MO no solo pode promover aumento da sorção dos agrotóxicos e seus metabólitos, influenciando sua extração (PERES et al., 2002).

Os inseticidas tiametoxam, permetrina e bifentrina (Figura 1) têm sido comumente usados para o controle de insetos-praga na cultura da soja, sendo aplicados no solo e/ou parte aérea das plantas, como também no tratamento de sementes (BRASIL, 2013). O tiametoxam pertence à classe dos neonicotinoides, apresenta solubilidade em água de $4.100 \mathrm{mg} \mathrm{L}^{-1}, \mathrm{~K}_{\mathrm{oc}}$ de $70 \mathrm{~mL} \mathrm{~g}^{-1} \mathrm{e}$ alto potencial de lixiviação no solo (CASTRO, 2005). A bifentrina e a permetrina são compostos do grupo químico dos piretroides, com alta toxicidade para organismos aquáticos, solubilidades de 0,001 e $0,2 \mathrm{mg} \mathrm{L}^{-1}$, respectivamente, $\mathrm{K}_{\mathrm{oc}}$ maiores que $100.000 \mathrm{~mL} \mathrm{~g}^{-1}$ e baixo potencial de lixiviação nos solos.

O objetivo do presente trabalho foi desenvolver e validar método analítico para a quantificação de resíduos dos inseticidas tiametoxam, permetrina e bifentrina em solo sob diferentes sistemas de manejo e, portanto, diferentes teores de MO, empregando-se CLAE.

\section{MATERIAL E MÉTODOS}

\subsection{PADRÕES ANALÍTICOS, REAGENTES E EQUIPAMENTOS}

As soluções analíticas estoque dos inseticidas tiametoxam, permetrina e bifentrina, na concentração de $1 \mathrm{mg} \mathrm{mL}^{-1}$, foram preparadas dissolvendo-se $10 \mathrm{mg}$ de cada padrão, separadamente, em $10 \mathrm{~mL}$ de acetona e armazenadas individualmente à temperatura de $-20^{\circ} \mathrm{C}$. Os padrões analíticos de tiametoxam, permetrina e bifentrina, com purezas de 99, 94 e $97 \%$ respectivamente, foram adquiridos de Dr. Ehrenstorfer (Alemanha) e FMC (Brasil). A partir das soluções estoque foram feitas soluções padrão por diluição em acetonitrila nas concentrações de 0,$5 ; 1,0 ; 1,5 ; 2,0$; $5,0 \mu \mathrm{g} \mathrm{mL}^{-1}$ para bifentrina, permetrina e tiametoxam, utilizadas para a construção das curvas analíticas. Também a partir das soluções estoque obteve-se a solução de trabalho, na concentração de $50 \mu \mathrm{g} \mathrm{mL}^{-1} \mathrm{em}$ acetona, utilizada para as fortificações das amostras de solo. 
<smiles>CN1COCN(Cc2cnc(Cl)s2)/C1=N\[O+]([O-])[O-]</smiles><smiles>CC1C(C=C(Cl)Cl)C1(C)C(=O)OCc1cccc(Oc2ccccc2)c1</smiles>

Permetrina<smiles>Cc1c(COC(=O)C(C)C(F)(F)F)cccc1-c1ccccc1</smiles><smiles>CC(Cl)=CC1C(C)C1C(=O)OCc1cccc(-c2ccccc2)c1C</smiles>

(Z)-(1S)-cis-

Bifentrina

\section{FIGURA 1 - ESTRUTURAS QUÍMICAS DO TIAMETOXAM, PERMETRINA E BIFENTRINA}

Utilizaram-se nos procedimentos de extração acetona da Carlo Erba (França), metanol grau HPLC da J.T. Baker (EUA) e diclorometano da Merck (Alemanha). Na purificação das amostras foram utilizadas sílica gel 60 da Merck (Alemanha) com granulometria na faixa de 0,040 a 0,063 mm, sulfato de sódio anidro e acetato de etila da Carlo Erba (França), acetonitrila grau HPLC e hexano da J.T. Baker (EUA). Toda vidraria utilizada foi previamente lavada com solução de água com detergente neutro a $1 \%$ e acetona da Qhemis (Brasil). Para a fase móvel, utilizou-se acetonitrila grau HPLC da J.T. Baker (EUA) e água ultrapura obtida pelo sistema da Gehaka (Brasil) com condutividade de $0,05 \mu \mathrm{S} \mathrm{cm} \mathrm{cm}^{-1}$ e resistividade de $18 \mathrm{~m} \Omega$. Na extração dos inseticidas utilizou-se mesa agitadora da Cientec (Brasil) operada a 216 rpm, banho ultrassônico da Unique (Brasil), e evaporador rotativo da Buchi (Suíça) com refrigerador de líquido.

\subsection{COLETA E PREPARO DAS AMOSTRAS DE SOLO}

As amostras de solo foram coletadas no campo experimental da Embrapa Agropecuária 


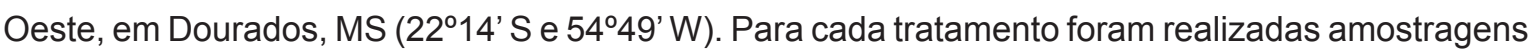
na profundidade de $0-10 \mathrm{~cm}$, sendo coletada amostra composta, proveniente de 50 subamostras retiradas de forma aleatória em cada parcela. O solo é classificado como Latossolo Vermelho distroférrico típico de textura argilosa (SANTOS et al., 2006). Os tratamentos compreenderam os seguintes sistemas de manejo: Integrado Lavoura-Pecuária (SILP), Plantio Direto (SPD) e Convencional (SC). Os principais atributos do solo para caracterização dos três tratamentos são mostrados na Tabela 1. Os solos provenientes dos diferentes sistemas são contrastantes com relação ao teor de MO, variando de 32,8 a $61,5 \mathrm{~g} \mathrm{dm}^{-3}$. Após a coleta, as amostras foram secas à sombra, destorroadas e peneiradas em malha de $2 \mathrm{~mm}$.

\section{TABELA 1 - ATRIBUTOS QUÍMICOS E FÍSICOS DO SOLO NA PROFUNDIDADE DE 0-10 cm SOB SISTEMA INTEGRADO LAVOURA-PECUÁRIA (SILP), SISTEMA PLANTIO DIRETO (SPD) E SISTEMA CONVENCIONAL (SC)}

\begin{tabular}{|c|c|c|c|c|c|c|}
\hline Tratamentos & $\begin{array}{c}\mathrm{pH} \\
\left(\mathrm{H}_{2} \mathrm{O}\right)\end{array}$ & $\begin{array}{c}\mathrm{pH} \\
\left(\mathrm{CaCl}_{2}\right)\end{array}$ & $\mathrm{MO}\left(\mathrm{g} \mathrm{dm}^{-3}\right)$ & Areia $\left(\mathrm{g} \mathrm{kg}^{-1}\right)$ & Silte $\left(\mathrm{g} \mathrm{kg}^{-1}\right)$ & Argila $\left(\mathrm{g} \mathrm{kg}^{-1}\right)$ \\
\hline SILP & 6,1 & 5,4 & 61,5 & 177 & 183 & 640 \\
\hline SPD & 6,3 & 5,7 & 44,7 & 177 & 167 & 656 \\
\hline SC & 5,6 & 4,9 & 32,8 & 160 & 167 & 673 \\
\hline
\end{tabular}

MO = Matéria Orgânica

\subsection{CONDIÇÕES CROMATOGRÁFICAS E AVALIAÇÃO DOS PARÂMETROS INSTRUMENTAIS}

Para identificação e quantificação dos inseticidas utilizou-se Cromatógrafo a líquido, modelo Varian 920-LC, equipado com coluna de fase reversa C-18 Polaris $(25 \mathrm{~cm} \times 4,6 \mathrm{~mm} \times$ $5 \mu \mathrm{m})$, pré-coluna C-18 Polaris $(2,5 \mathrm{~cm} \times 4,6 \mathrm{~mm} \times 5 \mu \mathrm{m})$ e detector de arranjo de diodos (DAD).

A quantificação do tiametoxam ocorreu por eluição em gradiente com: $50 \%$ acetonitrila e $50 \%$ água ultrapura v/v de 0 a 6 min; $100 \%$ acetonitrila de 6 a 10 min e $50 \%$ acetonitrila e $50 \%$ água ultrapura v/v de 10 a $20 \mathrm{~min}$, com fluxo de $1 \mathrm{~mL} \mathrm{~min}^{-1}$ e volume de injeção de $20 \mu \mathrm{L}$. A temperatura do forno da coluna foi de $35^{\circ} \mathrm{C}$ e o comprimento de onda de leitura das amostras de $254 \mathrm{~nm}$ (Figura 2), com tempo de retenção do tiametoxam de 3,48 min (Tabela 2 e Figura 3).

As condições para quantificação da permetrina e bifentrina por eluição em gradiente foram: $70 \%$ acetonitrila e $30 \%$ água ultrapura v/v de 0 a $5 \mathrm{~min} ; 100 \%$ acetonitrila de 5 a $15 \mathrm{~min}$ e $70 \%$ acetonitrila e $30 \%$ água ultrapura v/v de 15 a $22 \mathrm{~min}$, com fluxo de $1 \mathrm{~mL} \mathrm{~min}{ }^{-1}$ e volume de injeção de $20 \mu \mathrm{L}$. A temperatura do forno da coluna foi de $35^{\circ} \mathrm{C}$ e o comprimento de onda de leitura das amostras de $212 \mathrm{~nm}$ (Figura 2). Os tempos de retenção da bifentrina e permetrina I e II, nessas condições, foram de 12,37 min e 11,80 e 12,07 min, respectivamente (Tabela 2 e Figura 3).

A quantificação dos compostos ocorreu por meio da comparação das áreas dos picos das amostras com a curva de calibração, obtida mediante as injeções dos padrões analíticos.

\subsection{TESTE DE EFICIÊNCIA DE SOLVENTES E MÉTODOS DE EXTRAÇÃO}

Avaliou-se a eficiência de diferentes solventes (diclorometano + acetona (1:1) e metanol) e 
métodos de extração (mesa agitadora por 1 hora - M1; mesa agitadora por três horas - M3; mesa agitadora por 1 hora e ultrassom por 15 min - M1+U15; mesa agitadora por 1 hora e ultrassom por 30 min - M1+U30; mesa agitadora por 3 horas e ultrassom por 15 min - M3+U15; mesa agitadora por 3 horas e ultrassom por 30 min - M3+U30) na recuperação dos três inseticidas. Para tal, amostras de solo $(50 \mathrm{~g})$ do tratamento SILP foram transferidas para erlenmeyers com tampa e fortificadas com 1,0 $\mathrm{g} \mathrm{g} \mathrm{g}^{-1}$ de bifentrina, permetrina e tiametoxam. Em seguida, foram adicionados $150 \mathrm{~mL} \mathrm{de}$ cada solvente e/ou mistura aplicando-se os diferentes métodos de extração. Após essa etapa, as amostras foram colocadas em repouso (decantação) por 1 hora. Parte da fração líquida $(30 \mathrm{~mL}$ ) foi retirada e concentrada em evaporador rotativo até total secura. Na etapa de purificação dos extratos utilizou-se método adaptado de Pessini (2008), que consistiu na retomada dos inseticidas de cada amostra com três lavagens sucessivas usando-se $2 \mathrm{~mL}$ da mistura hexano/acetato de etila (1:1). Transferiu-se a fração da lavagem para coluna de sílica $(2 \mathrm{~g})$ mediante seringa de $10 \mathrm{~mL} \mathrm{com}$ frits PTFE e $0,5 \mathrm{~g}$ de sulfato de sódio anidro. Para a primeira eluição da coluna foram adicionados, por quatro vezes, $5 \mathrm{~mL}$ da mistura hexano/acetato de etila (1:1) para coleta da fração contendo os inseticidas permetrina e bifentrina. Na segunda eluição foram adicionados, por quatro vezes, $5 \mathrm{~mL}$ da mistura hexano/acetato de etila (1:1), sendo essa fração descartada. Após adição de $13 \mathrm{~mL}$ de acetonitrila coletou-se a fração do inseticida tiametoxam. As duas frações coletadas contendo os resíduos de permetrina+bifentrina e tiametoxam foram concentradas em evaporador rotativo até total secura e retomadas com $5 \mathrm{~mL}$ de acetonitrila. As amostras foram filtradas em filtro PTFE de $0,22 \mu \mathrm{m}$ e armazenadas diretamente em frascos a $-20^{\circ} \mathrm{C}$ até a injeção no cromatógrafo. Todas as análises foram efetuadas em triplicata.

Conduziu-se o experimento para avaliar a eficiência de solventes e métodos de extração utilizando delineamento inteiramente casualizado, em esquema fatorial $2 \times 6$ (duas soluções extratoras e seis métodos de extração) com três repetições. Os dados referentes às porcentagens de recuperação dos três inseticidas foram submetidos à análise de variância utilizando-se o programa R (R FOUNDATION..., 2012), e suas médias comparadas pelo teste t a $5 \%$.

Após a definição do solvente e método de extração mais eficiente, realizou-se o segundo teste para validar o método desenvolvido para determinação de permetrina, bifentrina e tiametoxam nos solos dos diferentes sistemas de manejo após a fortificação das amostras com diferentes concentrações $\left(0,25 ; 0,5 ; 1,0 \mu \mathrm{g} \mathrm{g}^{-1}\right)$. Os procedimentos de extração, purificação e quantificação para os três inseticidas foram os mesmos descritos anteriormente.

Os parâmetros de validação determinados foram: linearidade, seletividade, exatidão (recuperação), precisão (coeficiente de variação), limites de detecção (LD) e quantificação (LQ) do método e robustez. Avaliou-se a robustez do método pela sua aplicação aos solos com diferentes propriedades químicas, em particular diferentes teores de matéria orgânica, uma vez que esse é um dos principais fatores que podem influenciar a extração dos agrotóxicos em solos. Os LD e LQ do método para os compostos analisados foram determinados pelo procedimento de Thier e Zeumer (1987). Considerou-se como LQ o menor nível de fortificação que obedecesse, simultaneamente, recuperação superior a $70 \%$, fosse superior ao LD e apresentasse coeficiente de variação menor que $20 \%$.

\section{RESULTADOS E DISCUSSÃO}

Com base no espectro de absorção, os comprimentos de onda de máxima absorção $\left(\lambda_{\text {máx }}\right)$ para bifentrina e permetrina I e II foram de $212 \mathrm{~nm}$ e de $254 \mathrm{~nm}$ para o tiametoxam (Figura 2). O valor $\lambda_{\text {máx }}$ para bifentrina e permetrina de $212 \mathrm{~nm}$ enquadrou-se no intervalo de 204 a $220 \mathrm{~nm}$ relatados em vários estudos sobre a quantificação de resíduos em amostras de solo, água e tecido vegetal (BASKARAN, KOOKANA NAIDU, 1999; SILVÉRIO et al., 2012). Para o tiametoxam, o valor $\lambda_{\text {máx }}$ igual a $254 \mathrm{~nm}$ mostrou-se semelhante aos relatados por Scorza Júnior \& Rigitano (2012). 


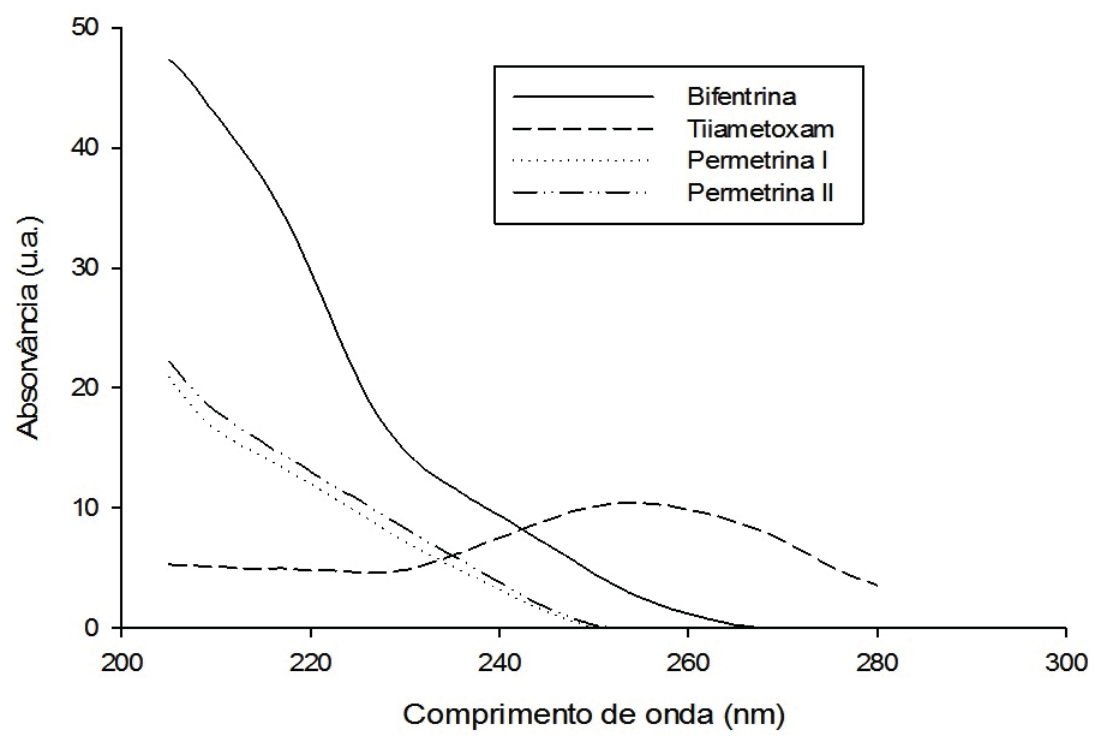

FIGURA 2 - ESPECTRO DE ABSORÇÃO DA ANÁLISE POR CLAE DA SOLUÇÃO DE BIFENTRINA, PERMETRINA I E II E TIAMETOXAM NA CONCENTRAÇÃO DE 2,0 $\mu \mathrm{g} \mathrm{ML-1EM} \mathrm{ACETONITRILA}$

Para todos os inseticidas avaliados, a faixa linear de concentração ficou entre 0,5 e $5,0 \mu \mathrm{g} \mathrm{mL}-1$, com valores do coeficiente de determinação $\left(R^{2}\right)$ superiores a 0,99 (Tabela 2 ).

TABELA 2 - PARÂMETROS ANALÍTICOS DE VALIDAÇÃO DO MÉTODO

\begin{tabular}{c|c|c|c|c|c}
\hline Inseticidas & TR (min.) & Faixa linear $\left(\boldsymbol{\mu g} \mathbf{~ m L}^{-1}\right)$ & $\mathbf{R}^{2}$ & $\mathbf{L D}^{*}\left(\boldsymbol{\mu g} \mathbf{~ g}^{-1}\right)$ & $\mathbf{L Q}^{* *}\left(\boldsymbol{\mu g ~ g}^{-1}\right)$ \\
\hline Tiametoxam & 3,48 & $0,5-5,0$ & 0,9996 & 0,07 & 0,25 \\
Permetrina I e II & $11,80 / 12,07$ & $0,5-5,0$ & 0,9994 & 0,05 & 0,25 \\
Bifentrina & 12,37 & $0,5-5,0$ & 0,9996 & 0,05 & 0,25 \\
\hline
\end{tabular}

*LD = limite de detecção do método; ** ${ }^{*} Q$ = limite de quantificação do método.

Os cromatogramas das amostras do branco, padrões analíticos e das amostras fortificadas durante o teste de recuperação dos diferentes inseticidas avaliados no solo sob SILP são mostrados na Figura 3. Observa-se que os cromatogramas das amostras do branco (Figura 3A e 3D) são isentos de picos nos tempos de retenção dos analitos avaliados, mostrando ausência de possíveis interferentes e, portanto, a boa seletividade do método. Nas amostras do branco sob SPD e SC também não foram observados picos nos tempos de retenção dos analitos avaliados.

Os diferentes métodos e solventes testados na extração de permetrina e bifentrina não apresentaram diferenças significativas $(p>0,05)$ (Tabela 3 ). Isso indica que todos os métodos (M1: Mesa agitadora por 1 hora; M3: mesa agitadora por 3 horas; M1+U15: mesa agitadora por 1 hora e ultrassom por 15 minutos; M1+U30: mesa agitadora por 1 hora e ultrassom por 30 minutos; M3+U15: mesa agitadora por 3 horas e ultrassom por 15 minutos; M3+U30: mesa agitadora por 3 horas e ultrassom por 30 minutos) e solventes (acetona + diclorometano (1:1) e metanol) avaliados na extração dos inseticidas foram eficientes, com recuperação para permetrina entre 94,5 e 
$108,3 \%$ e bifentrina entre 86,1 e $98,1 \%$. Para ambos os inseticidas, os valores do coeficiente de variação foram inferiores a $14 \%$. Os solventes mais usados na extração de piretroides tem sido hexano, benzeno e acetona ou a mistura binária de solventes, tal como acetona + hexano, diclorometano + hexano, ou isooctano + acetona (ALI e BAUGH, 2003; LUO, SHAO e ZHANG, 2010). Além desses solventes, o uso de ultrassom e mesa agitadora têm apresentado resultados satisfatórios ao processo de extração simultânea desses inseticidas no solo (YASIN et al., 1996). Soares (2011) validando método de extração com mesa agitadora e banho em ultrassom para diferentes inseticidas, incluindo bifentrina e tiametoxam em amostras de uva, mostrou que os dois métodos são eficientes nas recuperações. No entanto, a mesa agitadora proporcionou melhor precisão dos resultados.

Para o inseticida tiametoxam, os diferentes métodos testados não mostraram diferença estatística $(p>0,05)$ quando conduzidos com solução extratora acetona + diclorometano (1:1) ou metanol (Tabela 3). Assim, os diferentes métodos avaliados foram eficientes para extração desse inseticida no solo, com valores de recuperação entre 89,6 e 99,3 \% para acetona + diclorometano (1:1) e entre 71,4 e $83,0 \%$ para metanol. Os valores do coeficiente de variação foram inferiores a $13 \%$ para ambas as soluções extratoras e diferentes métodos.

Amostra do branco (A e D), amostra com padrão analítico na concentração de $2,0 \mu \mathrm{g} \mathrm{mL}^{-1}$ (B e E) e amostra fortificada com 2,0 $\mu \mathrm{g} \mathrm{mL}^{-1}$ durante o teste de recuperação (C e F).

Várias técnicas têm sido propostas para a extração de agrotóxicos no solo, como o uso da mesa agitadora com a amostra do solo colocada sob agitação em diferentes tempos conforme as características do solo e do analito (GUARDINO et al., 1998; BOSSI, VEJRUP e JACOBSEN., 1999). As técnicas modernas têm apresentado outros métodos de extração como banho de ultrassom, sonda de ultrassom e extração acelerada com solventes seguido de purificação das amostras (KORN, PEREIRA e BORGES, 2005; RAMOS et al., 2010). A redução do tempo da extração, do volume de reagentes e do custo das análises têm sido evidenciada com o uso desses métodos (BARBOZA e SERRA, 1992). Yasin et al. (1996), avaliando método para análise e determinação simultânea de cinco inseticidas piretroides em solo, obtiveram recuperação dos compostos entre 81,7 e 108,2 \% com esse método para a extração dos analitos. No entanto, o uso do ultrassom na extração do inseticida tiametoxam em tubérculos e solo cultivado com batata apresentou menor recuperação quando comparado ao o uso da mesa agitadora (BITTENCOURT, 2008). Nascentes et al. (2002) relataram que o uso do banho de ultrassom pode influenciar negativamente a recuperação dos agrotóxicos, tendo como possíveis causas a posição das amostras no ultrassom, o volume e a temperatura da água do banho. De maneira geral, os novos métodos buscam processos de extração dos agrotóxicos do solo com maior eficiência, redução de custos e do tempo das análises (PICÓ et al., 2007). No entanto, em muitas situações, há necessidade de priorizar apenas uma dessas vantagens em detrimento das outras.

$\mathrm{O}$ método $\mathrm{M} 1$, eficiente para a recuperação dos três inseticidas avaliados com economia de tempo, foi selecionado para a extração dos inseticidas no solo. Considerando o método M1, a solução extratora acetona + diclorometano $(1: 1)$ foi estatisticamente superior $(p<0,05)$ ao metanol para extração do tiametoxam (Tabela 3 ), sendo portanto selecionada como solução extratora no presente trabalho.

Após a definição do método e da solução extratora mais eficiente procedeu-se ao estudo de recuperação, utilizando diferentes solos e concentrações. O método de extração adotado apresentou recuperações eficientes para o solo dos diferentes sistemas de manejo e concentrações avaliadas (Tabela 4), com valores variando de 88,3 a 105,5\% para a bifentrina, 88,2 a 103,0 \% para a permetrina e de 74,8 a $94,7 \%$ para o tiametoxam. Já os valores dos coeficientes de variação foram de 1,0 a 13,7 \% para todos os inseticidas, solos e concentrações avaliadas. A eficiência da extração de agrotóxicos está fortemente relacionada às características físicas e químicas do solo (PERES et al., 2002). Resíduos vegetais de culturas anteriores dos diferentes sistemas de manejo e sua decomposição por micro-organismos do solo influenciam os padrões de acúmulo de matéria 
orgânica e pode favorecer o processo de adsorção dos agrotóxicos (SILVA e FAY, 2004; LOPES et al., 2002). Correia et al. (2008), avaliando a influência de diferentes sistemas de manejo sobre o potencial de adsorção de atrazina, mostraram que a quantidade de $\mathrm{MO}$ e sua qualidade em plantio direto favorecem a adsorção desse composto no solo. Luchini (1987) relatou que o teor de MO e argila do solo podem adsorver fortemente o agrotóxico, dificultando a extração dos analitos da matriz.

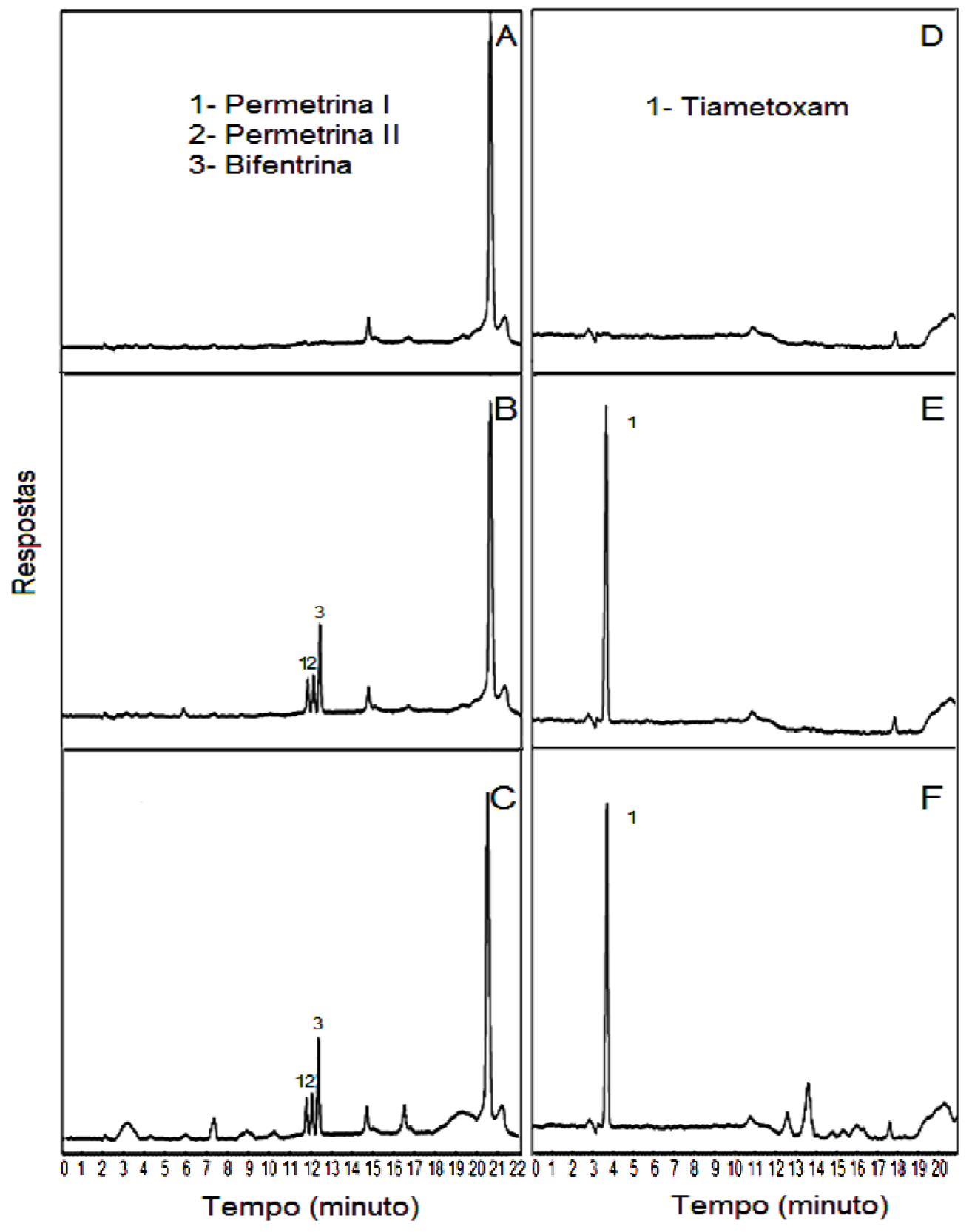

FIGURA 3 - CROMATOGRAMAS DA ANÁLISE POR CLAE DE PERMETRINA (I E II), BIFENTRINA E TIAMETOXAM

Amostra do branco ( $A$ e D), amostra com padrão analítico na concentração de $2,0 \mu \mathrm{g} \mathrm{mL}^{-1}$ (B e E) e amostra fortificada com $2,0 \mu \mathrm{g} \mathrm{mL}^{-1}$ durante o teste de recuperação (C e F).

Os resultados enfatizam a eficiência do método adotado com mesa agitadora 1 hora (M1) e a solução extratora acetona + diclorometano (1:1) nas diferentes concentrações e sistemas de manejo avaliados, mostrando valores de recuperação dentro da faixa de aceitação de 70 a 120 \% 
e coeficiente de variação até $20 \%$ (RIBANI et al., 2004). O método se mostrou robusto já que não apresentou variações significativas na precisão e exatidão para os diferentes solos.

\section{TABELA 3 - RECUPERAÇÃO DE PERMETRINA, BIFENTRINA E TIAMETOXAM EM AMOSTRAS DE SOLO SOB O SISTEMA INTEGRADO LAVOURA-PECUÁRIA COM DIFERENTES SOLUÇÕES EXTRATORAS E MÉTODOS}

\begin{tabular}{|c|c|c|c|}
\hline \multirow{2}{*}{ Inseticidas } & \multirow{2}{*}{ Método } & \multicolumn{2}{|c|}{ Solução extratora } \\
\hline & & Acetona/ diclorometano $(1: 1)$ & Metanol \\
\hline \multirow{6}{*}{ Tiametoxam } & M1 & $89,6 \pm 3,7(7,3) \quad a$ & $72,7 \pm 0,5(1,4) b$ \\
\hline & M3 & $99,3 \pm 3,4(6,0) \quad a$ & $83,0 \pm 4,1(8,7) b$ \\
\hline & $\mathrm{M} 1+\mathrm{U} 15$ & $97,1 \pm 6,9(12,5) a$ & $71,4 \pm 0,8(2,1) b$ \\
\hline & $\mathrm{M} 1+\mathrm{U} 30$ & $90,2 \pm 0,9(1,8)$ a & $72,3 \pm 1,0(2,7) b$ \\
\hline & M3+U15 & $98,8 \pm 0,5(0,9) \quad a$ & $72,2 \pm 0,8(1,9) b$ \\
\hline & $\mathrm{M} 3+\mathrm{U} 30$ & $97,2 \pm 1,2(2,2) \quad a$ & $72,2 \pm 0,6(1,6) b$ \\
\hline \multirow{6}{*}{ Permetrina } & M1 & $100,5 \pm 4,1(7,1)$ & $102,8 \pm 0,8(1,5)$ \\
\hline & M3 & $102,5 \pm 4,3(7,3)$ & $105,0 \pm 2,5(4,3)$ \\
\hline & $\mathrm{M} 1+\mathrm{U} 15$ & $100,1 \pm 3,6(6,3)$ & $98,8 \pm 3,1(5,4)$ \\
\hline & $\mathrm{M} 1+\mathrm{U} 30$ & $98,0 \pm 1,4(2,6)$ & $96,1 \pm 1,6(3,0)$ \\
\hline & $\mathrm{M} 3+\mathrm{U} 15$ & $94,5 \pm 2,4(4,5)$ & $102,6 \pm 2,7(4,7)$ \\
\hline & $\mathrm{M} 3+\mathrm{U} 30$ & $96,4 \pm 0,9(1,6)$ & $97,7 \pm 0,9(1,7)$ \\
\hline \multirow{6}{*}{ Bifentrina } & M1 & $92,5 \pm 4,5(8,5)$ & $90,4 \pm 2,3(4,4)$ \\
\hline & M3 & $93,1 \pm 3,9(7,4)$ & $93,2 \pm 2,8(5,4)$ \\
\hline & $\mathrm{M} 1+\mathrm{U} 15$ & $86,1 \pm 1,0(2,2)$ & $93,8 \pm 3,2(5,9)$ \\
\hline & $\mathrm{M} 1+\mathrm{U} 30$ & $88,9 \pm 1,3(2,5)$ & $88,6 \pm 2,9(5,7)$ \\
\hline & $\mathrm{M} 3+\mathrm{U} 15$ & $86,0 \pm 1,1(2,3)$ & $98,1 \pm 1,9(3,4)$ \\
\hline & $\mathrm{M} 3+\mathrm{U} 30$ & $88,7 \pm 2,8(5,5)$ & $89,2 \pm 1,3(2,6)$ \\
\hline
\end{tabular}

*Média \pm erro padrão. **Valores entre parênteses representam o coeficiente de variação (\%). Médias seguidas pelas mesmas letras minúsculas na linha são estatisticamente semelhantes pelo teste t a $5 \%$. $(n=3)$. Amostras fortificadas com $1,0\left(\mu g g^{-1}\right)$. M1: Mesa agitadora por 1 hora; M3: mesa agitadora por 3 horas; M1+U15: mesa agitadora por 1 hora e ultrassom 15 minutos; M1+U30: mesa agitadora por 1 hora e ultrassom por 30 minutos; M3+U15: mesa agitadora por 3 horas e ultrassom por 15 minutos; M3+U30: mesa agitadora por 3 horas e ultrassom por 30 minutos.

As metodologias de análise de piretroides em solo preconizam de modo geral o uso da cromatografia a gás com detector por captura de elétrons ou acoplada a espectrometria de massas, que normalmente fornecem limites de detecção uma ou duas ordens de grandeza menores que os aqui determinados (SHARMA e SINGH, 2012). Entretanto, a vantagem desse método reside 
na detecção simultânea dos inseticidas piretroides bifentrina e permetrina com o neonicotinoide tiametoxam, que só pode ser analisado por cromatografia a líquido (GUPTA, GAJBHIYE e GUPTA, 2012).

\section{TABELA 4 - PRECISÃO E EXATIDÃO DE RECUPERAÇÃO DO TIAMETOXAM, PERMETRINA E BIFENTRINA EM SOLO COM DIFERENTES SISTEMAS DE MANEJO: INTEGRADO LAVOURA- PECUÁRIA (SILP), SISTEMA PLANTIO DIRETO SPD) E SISTEMA CONVENCIONAL (SC)}

\begin{tabular}{|c|c|c|c|c|}
\hline \multirow{3}{*}{ Inseticidas } & \multirow{3}{*}{$\begin{array}{c}\text { Concentração } \\
\left(\mu \mathrm{g} \mathrm{g}^{-1}\right)\end{array}$} & \multicolumn{3}{|c|}{ Sistemas de manejo } \\
\hline & & SILP & SPD & SC \\
\hline & & \multicolumn{3}{|c|}{ Recuperação média (n=3) (\%) } \\
\hline \multirow{3}{*}{ Tiametoxam } & 1,0 & *89,6 $\pm 3,7(7,3)$ & $84,2 \pm 2,9(6,1)$ & $94,7 \pm 1,5(2,7)$ \\
\hline & 0,5 & $85,5 \pm 6,8(13,7)$ & $74,8 \pm 1,7(4,0)$ & $89,1 \pm 3,9(7,6)$ \\
\hline & 0,25 & $75,3 \pm 3,9(9,1)$ & $77,2 \pm 3,7(8,5)$ & $77,9 \pm 2,0(4,7)$ \\
\hline \multirow{3}{*}{ Permetrina } & 1,0 & $100,5 \pm 4,1(7,1)$ & $90,4 \pm 3,3(6,4)$ & $103,0 \pm 3,2(5,4)$ \\
\hline & 0,5 & $101,7 \pm 6,2(10,7)$ & $101,3 \pm 5,3(9,1)$ & $98,9 \pm 5,4(9,7)$ \\
\hline & 0,25 & $88,2 \pm 2,4(4,8)$ & $100,0 \pm 0,5(1,0)$ & $92,7 \pm 3,9(7,4)$ \\
\hline \multirow{3}{*}{ Bifentrina } & 1,0 & $92,5 \pm 4,2(8,5)$ & $88,3 \pm 3,0(6,0)$ & $90,6 \pm 2,1(4,2)$ \\
\hline & 0,5 & $91,2 \pm 2,6(5,0)$ & $91,2 \pm 2,6(5,0)$ & $96,4 \pm 3,5(6,4)$ \\
\hline & 0,25 & $97,0 \pm 3,6(6,5)$ & $105,5 \pm 3,2(5,1)$ & $91,9 \pm 3,4(6,4)$ \\
\hline
\end{tabular}

*Média \pm erro padrão (coeficiente de variação).

\section{CONCLUSÃO}

O método proposto para extração e quantificação simultânea de resíduos de bifentrina, permetrina e tiametoxam em amostras de solo com diferentes teores de matéria orgânica mostrouse satisfatório com a utilização de mesa agitadora por 1 hora e solução extratora acetona: diclorometano (1:1). O método apresentado é robusto e rápido, tendo como peculiaridade a possibilidade da determinação simultânea de três inseticidas com polaridades diferenciadas. Para todos os inseticidas, o limite de quantificação do método analítico proposto foi de $0,25 \mathrm{\mu g} \mathrm{g}^{-1}$. Espera-se que esse método possa ser utilizado para análise de rotina em monitoramento de resíduos desses inseticidas em solos dos sistemas de produção de soja no Brasil.

\section{ABSTRACT}

\section{DETERMINATION OF THIAMETHOXAM, BIFENTHRIN AND PERMETHRIN INSECTICIDE RESIDUES IN SOIL SAMPLES}

Analytical methods for the quantification of residues of thiamethoxam, bifenthrin and permethrin in soil using high-performance liquid chromatography were developed and validated. Different methods and solvents for extraction of the three insecticides were tested in soils with a range of organic matter content between 32.8 and $81.7 \mathrm{~g} \mathrm{dm}^{-3}$. The method using shaking for one hour and acetone+dichloromethane (1:1) extraction solution proved to be the most suitable. Average recoveries were equal to $89.6 \%( \pm 3.7)$ for thiamethoxam, $100.5 \%( \pm 4.1)$ 
for permethrin and $92.5 \%( \pm 4.5)$ for bifenthrin, with variation coefficients lower than $14 \%$ for all insecticides. The limits of detection for thiamethoxam, bifenthrin and permethrin were $0.07,0.05$ and $0.05 \mathrm{\mu g} \mathrm{g}^{-1}$, respectively, and the limit of quantification for all insecticides was $0.25 \mu \mathrm{g} \mathrm{g}^{-1}$.

KEY-WORDS: HPLC; PESTICIDE RESIDUES; SOIL; THIAMETHOXAM; BIFENTHRIN; PERMETHRIN.

\section{REFERÊNCIAS}

1 ANDREU, V.; PICÓ Y. Determination of pesticides and their degradation products in soil: critical review and comparison of methods. Trends Anal. Chemical., v. 23, p. 772-789, 2004.

2 ALI, M.A.; BAUGH, P.J. Pyrethroid soil extraction, properties of mixed solvents and time profiles using GC/MS-NICl analysis. International Journal of Environmental Analytical Chemistry, v.83, n.11, p. 99-922, 2003.

3 BASKARAN, S.; KOOKANA, R.S.; NAIDU, R. Degradation of bifenthrin, chlorpyrifos and imidacloprid in soil and bedding materials at termiticidal application rates. Pesticide Science, v.55, p.1222-1228, 1999.

4 BARBOZA, J.C.S.; SERRA, A.A. Ultra-som(I): influência do ultra-som na química. Química Nova, v.15, n. 4, p. 302-316, 1992.

5 BITTENCOURT, L.M. Dissipação e monitoramento dos inseticidas clorpirifós e thiamethoxam em tubérculos e solo cultivado com batata (Solanum tuberosum L.). 2008. 93 p. Dissertação (Mestrado em Agroquímica). Universidade Federal de Viçosa, Viçosa, 2008.

6 BOSSI, R.; VEJRUP, K.; JACOBSEN, C. S. Determination of sulfonylurea degradation products in soil by liquid chromatography-ultraviolet detection followed by confirmatory liquid chromatography-tandem mass spectrometry. Journal of Chromatography A, v. 855, n.2, p. 575-582, 1999.

7 BRASIL. Ministério da Agricultura, Pecuária e Abastecimento. Agrofit. [Brasília, DF], 2003. Disponível em: http :// extranet. agricultura.gov.br/agrofit_cons/principal_agrofit_cons. Acesso em: 20 de Julho 2013.

8 CASTRO, N.R.A. Sorção, degradação e lixiviação do inseticida thiamethoxam em latossolo e argissolo. 2005. 161 p. Tese (Doutorado em Agronomia - área Entomologia), Universidade Federal de Lavras, Lavras, 2005.

9 CHAIM, A.; VALARINI, P.J.; OLIVEIRA, D.A.; MORSOLETO, R.V.; PIO, L.C. Avaliação de perdas de pulverização em culturas de feijão e tomate. Jaguariúna: Embrapa Meio Ambiente, 1999. 29 p. (Embrapa Meio Ambiente. Boletim de Pesquisa, 2).

10 CORREIA, F.V.; MERCANTE, F.M.; FABRÍCIO, A.C.; CAMPOS, T.M.P.; VARGAS JR., E.; LANGENBACH, T. Adsorção de atrazina em solo tropical sob plantio direto e convencional. Pesticidas: revista de ecotoxicologia e meio ambiente, v.17, p.37-46, 2008.

11 GONÇALVES, C.; ALPENDURADA, M.F. Assessment of pesticide contamination in soil samples from an intensive horticulture area, using ultrasonic extraction and gas chromatography-mass spectrometry, Talanta, v.65, n.5, p.11791189, 2005.

12 GUPTA, S.; GAJBHIYE, V.T.; GUPTA, R.K. Soil dissipation and leaching behavior of a neonicotinoid insecticide thiamethoxam. Bulletin of Environmental Contamination and Toxicology, v.80, n.5, p.431-437, 2008.

13 GUARDINO, X.; OBIOLS, J.; ROSSEL, M.; G. FARRAH, A.; SERRA, C. Determination of chlorpyrifos in air, leaves and soil from a greenhouse by gas chromatography with nitrogen-phosphorus detection, high-performance liquid chromatography and capillary electrophoresis. Journal of Chromatography A, Amsterdam, v.823, n.1, p.91-96, 1998.

14 KORN, M.; PEREIRA, M.G.; BORGES, S.S. Algumas aplicações analíticas dos ultra-sons. Boletim da Sociedade Portuguesa de Química, v.96, p.51-56, 2005.

15 LANÇAS, F.M. Cromatografia líquida moderna (HPLC/CLAE). Campinas (SP): Átomo, 2009. 382 p.

16 LEHOTAY, S.J.; KOK, A.; HIEMSTRA, M.; BODEGRAVEN, P.V. Validation of a fast and easy method for the determination of residues from 229 pesticides in fruits and vegetables using gas and liquid chromatography and mass spectrometric detection. Journal of AOAC International, v.88, p.595-614, 2005.

17 LOPES, N.P.; QUEIROZ, M.E.R.; NEVES, A.A.; ZAMBOLIM, L. Influência da matéria orgânica na adsorção do fungicida triadimenol pelo solo. Química Nova, v.25, n.4, p.544-547, 2002.

18 LUCHINI, L. C. Adsorção-dessorção dos herbicidas paraquat, diuron e 2,4-D em seis solos brasileiros. 1987. $97 \mathrm{f}$. Dissertação (Mestrado em Energia Nuclear na Agricultura), Escola Superior de Agricultura Luiz de Queiroz , Piracicaba, 1987.

19 LUO, L.; SHAO, B.; ZHANG, J. Pressurized liquid extraction and cleanup procedure for the determination of pyrethroids 
in soils using gas chromatography/tandem mass spectrometry. Analytical Sciences, v. 26, p. 461-465, 2010.

20 MOREIRA, J.C.; PERES, F.; SIMÕES, A.C.; PIGNATI, W.A.; DORES, E.C.; VIEIRA, S.N.; STRÜSSMANN, C.; MOTT, T. Contaminação de águas superficiais e de chuva por agrotóxicos em uma região do estado do Mato Grosso. Ciência \& Saúde Coletiva, v.17, n.6, p.1557-1568, 2012.

21 NASCENTES, C.C.; KORN, M.; SOUSA, C.S.; ARRUDA, M.A.Z. Use of ultrasonic baths for analytical applications: a new approach for optimisation conditions. Journal of the Brazilian Chemical Society, v.12, p.57-63, 2001.

22 NOGUEIRA, E.N.; DORES, E.F.G.C.; PINTO, A.A.; AMORIM, R.S.S.; RIBEIRO, M.L.; LOURENCETTI, C. Currently used pesticides in water matrices in Central-Western Brazil. Journal of the Brazilian Chemistry Society, v.23, n.8, p.14761487,2012

23 PESSINI, M. M. O. Resíduos de acetamiprid e thiamethoxam em tomate estaqueado (Lycopersicon esculentum Mill.) em diferentes modalidades de aplicação. 2003. 88 p. Dissertação (Mestrado em Ciências - área Entomologia), Escola Superior de Agricultura Luiz de Queiroz, Piracicaba , 2003.

24 PERES, T.B.; PAPINI, S.; MARCHETTI, M.; NAKAGAWA, L.E.; MARCONDES, M. A.; ANDRÉA, M.M. ; LUCHINI, L.C. Métodos de extração de agrotóxicos de diversas matrizes. Arquivos do Instituto Biológico, v.69, n.4, p.87-94, 2002.

25 PICÓ, Y.; FERNÁNDEZ, M.; RUIZ, M.J.; FONT, G. Current trends in solid-phase-based extraction techniques for the determination of pesticides in food and environment. Journal Biochem. Biophys. Methods, v.70, p.117-31, 2007.

26 R Foundation for Statistical Computing. R: a language and environment for statistical computing. Vienna, 2012.

27 RAMOS, A.M.; BORGES, J.H.; PÉREZ, R.L.M.; DELGADO, R.M.A. Evaluation of a modifield QuECHERS method for the extraction of pesticides from agricultural, ornamental and forestal soils. Analytical and Bioanalytical Chemistry, v. 396, n.6, p. 2307-2319, 2010.

28 RIBANI, M.; BOTTOLI, C.B.G.; COLLINS, C.H.; JARDIM, I.C.S.F.; MELO, L.F.C. Validação em métodos cromatográficos e eletroforéticos. Química Nova, v.27, p.771-780, 2004.

29 SANTOS, H.G.; JACOMINE, P.K.T.; ANJOS, L.H.C.; OLIVEIRA, V.A.; OLIVEIRA, V.B.; COELHO, M.R.; LUMBREAS, J.F.; CUNHA, T.J.F. Sistema brasileiro de classificação de solos. 2. ed. Rio de Janeiro: EMBRAPA Solos, 2006. 306 p.

30 SCORZA JÚNIOR, R.P.; RIGITANO, R.L.O. Sorção, degradação e lixiviação do inseticida tiametoxam em dois solos de Mato Grosso do Sul. Revista Brasileira de Engenharia Agrícola e Ambiental, v.16, n.5, p.564-572, 2012.

31 SCHEYER, A.; GRAEFF, C.; MORVILLE, S., MIRABEL, P.; MILLET, M. Analysis of some organochlorine pesticides in an urban atmosphere (Strasbourg, east of France). Chemosphere, v.58, p.1517-1524, 2005.

32 SHARMA, D.; SINGH, S.B. Persistence of bifenthrin in sandy loam soil as affected by microbial community. Bulletin of Environmental Contamination and Toxicology, v.88, n.6, p.906-908, 2012.

33 SILVÉRIO, F.O.; SILVA, J.G.S.; AGUIAR, M.C.S.; CACIQUE, A.P.; PINHO, G. P. Análise de agrotóxicos em água usando extração líquido-líquido com partição em baixa temperatura por cromatografia líquida de alta eficiência. Química Nova, v.35, n.10, p.2052-2056, 2012.

34 SILVA, C.M.M.S.; FAY, E.F. Agrotóxicos e ambiente. Brasília, DF: Embrapa Informação Tecnológica, 2004. 400 p.

35 SOARES, C.E.S. Extração sólido-líquido com partição à baixa temperatura e seu emprego na análise de multirresíduos de agrotóxicos em uvas e derivados. 2011. 108 p. Dissertação (Mestrado em Agroquímica), Universidade Federal de Viçosa, Viçosa, 2011.

36 THIER, H.P.; ZEUMER, H. Manual of pesticide residue analysis. New York: VHC, 1987. 218 p.

37 YASIN, M.; BAUGHA, P.J.; BONWICKB, G.A.; DAVIESB, D.H.; HANCOCKA, P.; LEINOUDIA, M. Analytical method development for the determination of synthetic pyrethroid insecticides in soil by gas chromatography-mass spectrometry operated in negative-ion chemical-ionization mode. Journal of Chromatography A, v.754, p. 235-243, 1996.

\section{AGRADECIMENTOS}

Irzo Isaac Rosa Portilho agradece ao CNPq pela bolsa de estudo, ao curso de pós-graduação em Recursos Naturais e à Embrapa Agropecuária Oeste (Laboratório de Análises de Resíduos de Pesticidas) pelo apoio no desenvolvimento do trabalho. Ao laboratorista da Embrapa Agropecuária Oeste, Klerisson de Souza Duro, pelo apoio técnico na realização do trabalho. 